\title{
Analysis of Air Gap Permanent Magnet Eddy Current Coupling of the Equivalent Magnetic Charge Method Based on Field
}

\author{
Qingzhong Gao ${ }^{1,2}$, Dazhi Wang ${ }^{1}$ and Sheng Lin ${ }^{1,2}$ \\ ${ }^{I}$ College of Information Science and Engineering, Northeastern University, China \\ ${ }^{2}$ Academy of Automation, Shenyang Institute of Engineering, China \\ gaoqingzhong_xia@163.com
}

\begin{abstract}
This paper introduces the structure and working principle of permanent magnet eddy current coupling, using the equivalent magnetic charge principle to establish an analytical and mathematical model of permanent magnet eddy current coupling gap magnetic field, to solve the magnetic induction intensity. Changes in the parameters of gap size, the thickness of the magnetic blocks and magnetic block radius are obtained from using MATLAB to affect the air gap magnetic flux density's reflective curves in a single cycle. Through the analysis of the strength of the air gap magnetic induction, it lays the foundation for solving the transfer torque distribution of the eddy current density and magnetic.
\end{abstract}

Keywords: permanent magnet eddy current coupling; equivalent magnetic charge; air gap; magnetic induction intensity

\section{Introduction}

Permanent magnet eddy current coupling has been widely applied in the middle and high-power motor-driven pumps and fans and other equipment [1-5] and the device has characteristics such as high efficiency, low maintenance costs, simple installation, and high torque transmission, allowing the impact load, vibration isolation and flexible start and so on.

Permanent magnet eddy current coupling can be regarded as the improvement of permanent magnetic coupling[6-7] but the difference lies in that the structure of the two discs of the permanent magnetic couplings are both permanent, yet one disc of the permanent eddy current coupling is permanent and the other is a copper disc. As a non-contact coupling, it relies on air gap magnetic field passing power, torque, strong and weak magnetic field intensity distribution of the air gap directing an impact on copper coil eddy current density distribution and the size of the magnetic moment passed, so getting the air-gap magnetic field distribution is very meaningful and it is the foundation to solve accurate magnetic transfer torque.

\section{Structure and Working Principle of Permanent Magnet Eddy Current Couplings}

Mechanical structure of permanent magnet permanent magnet eddy current coupling [8-9] shown in Figure 1, is divided into two parts: one end of the connection conductor and motor rotor (copper plate); secondly, the permanent magnetic rotor connected to the load (permanent disk).Conductor rotor with permanent magnetic rotor can rotate independently and freely; when the motor is driven by rotation of the rotor conductor, the conductor plate is cutting line permanent magnetic rotor permanent magnets and eddy currents are generated in the conductor surface, thus forming the induced magnetic field; 
according to principle that the same opposites attract, coupling force is generated from the adjacent induced magnetic field to the permanent magnet, which is suction and repulsion between the adjacent induced magnetic field and the permanent magnet. These two forces are superposed in the rotating direction, which drives the permanent magnet rotor rotating in the same direction as the conductor of the rotor resulting in producing torque on the load side of the output shaft, so as to drive load rotational movement [10].

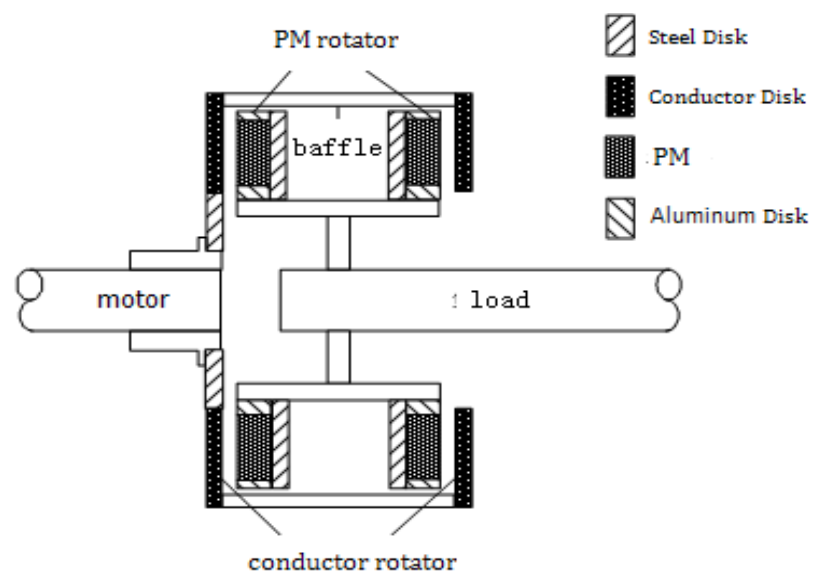

\section{Figure 1. Mechanical Structure of Adjustable Permanent Magnetic Coupler}

\section{The Calculation of Air Gap Field}

In fact, when a permanent magnet eddy current coupling is at work, the distribution of eddy current on the copper coil is pretty complex, which makes the calculation of eddy currents induced in the magnetic field strength is very difficult, so it is not a very effective method to calculate the eddy currents induced magnetic field. Thus, in the process of analyzing the strength of the permanent magnet in the air-gap magnetic field eddy current coupling, only consider the permanent magnet inducing a magnetic field strength, and ignore magnetic field strength induced by the eddy currents inside copper coil [11-13].

\subsection{The Air Gap Field Model Hypothesis}

Before analyze permanent magnet eddy current coupling model of air gap magnetic field, the following assumptions have been made[14-16]: (1)The permanent magnet can be regarded as closely aligned magnetic dipoles, after the permanent magnets are magnetized, it makes its magnetic dipole arrangement consistent with all permanent magnet material isotropic, homogeneous magnetization is magnetic medium, and the permanent magnet material is uniformly magnetized in the axial direction.(2)Just the magnetic field strength of permanent magnets are considered and copper coil inducing a magnetic field strength of the Eddy current effect could be ignored .(3) Wing disks of iron and copper coil liner are thick enough, so magnetic saturation phenomenon does not occur in the lining of the iron and it does not need to consider the impact of the magnetization of the iron lining gap magnetic field strength. Thickness (4) the thickness of the permanent magnet is far less than the radial length, ignoring the effect of the end portion of the permanent magnet.

\subsection{Mathematical Model}

What we can see from the equivalent magnetic charge method is when we calculate the permanent magnet magnetic field strength generated by a point in space, solving magnetic induction magnetic dipole generated in the unknown point is most important, and then 
obtaining the magnetic field strength at a certain point of the permanent magnet production. The total magnetic field point position leads to seeking expressions for $\mathrm{U}$ homogeneous isotropic permanent magnets inside each magnetic dipole is as follows [17-18]:

$$
U=\frac{1}{4 \pi \mu_{0}} \int_{v} \frac{\rho_{m}}{|\vec{r}|} d v+\frac{1}{4 \pi \mu_{0}} \int_{s} \frac{\sigma_{m}}{|\vec{r}|} d s
$$

Among above: $\rho_{m}$ is the equivalent body magnetic charge density; $\sigma_{m}$ is equivalent surface magnetic charge density; $\vec{r}$ is any source of permanent magnets to be seeking a vector field point, if the source is a positive magnetic charge, the vector $\vec{r}$ is from the source point to be seeking a positive magnetic charge field point. if the source is a negative magnetic charge, upon request by the vector $\vec{r}$ field point to the source point of the negative magnetic charge; $|\vec{r}|$ is any of the permanent magnet field source to the unknown point distance; $v$ is volume of permanent magnets; s stands for the boundary surface of the permanent magnet $\mu_{0}$ stands for the Permeability of vacuum In the derivation of the total magnetic bit, we can get the following expression of the equivalent surface magnetic charge density and equivalent magnetic charge density:

$$
\begin{gathered}
\rho_{m}=-\mu_{0} \nabla \cdot \vec{M} \\
\sigma_{m}=\mu_{0} \vec{M} \cdot \vec{n}
\end{gathered}
$$

Among above: $M$ stands for the inherent strength of $t$ the permanent magnet poles(residual magnetization) outer normal unit vector of the boundary surface of the permanent magnet. The assumptions (1) the permanent magnet materials uniformly magnetized in the axial direction, result in obtaining inherent strength of the permanent magnet poles $\vec{M}$ :

$$
\vec{M}=\frac{B_{r}}{\mu_{0}} \vec{e}_{z}
$$

Among above: $B_{r}$ stands for the residual magnetic flux density of the permanent magnet. $\vec{e}_{z}$ Stands for the permanent magnet axial unit vector. Assuming that (1) the permanent magnet material is isotropic, homogeneous magnetization is magnetic medium, the residual magnetic flux density of the permanent magnets $B_{r}$ is constant. Therefore, the (4) is substituted into (2) and (3) can prove that:

$$
\begin{gathered}
\rho_{m}=0 \\
\sigma_{m}=B_{r} e_{z} \cdot \vec{n}=\left\{\begin{array}{c}
B_{r} \\
-B_{r} \\
0
\end{array}\right.
\end{gathered}
$$

By the formula (5) and (6) we can draw that the equivalent body permanent magnet magnetic charge density $\rho_{m}$ is constant zero. Only the upper and lower end faces in the axial direction have a uniform distribution of magnetic charges. Residual magnetic flux density $\sigma_{m}$ is equal to the absolute value of the surface density of the permanent magnet $B_{r}$. Thus, we can simplify (1) into: 


$$
U=\frac{1}{4 \pi \mu_{0}} \int_{s} \frac{\sigma_{m}}{|\vec{r}|} d s=\frac{1}{4 \pi \mu_{0}} \int_{s} \frac{B_{r} \vec{e}_{z} \cdot \vec{n}}{|\vec{r}|} d s=\left\{\begin{array}{c}
\frac{1}{4 \pi \mu_{0}} \int_{s} \frac{B_{r}}{|\vec{r}|} d s \\
-\frac{1}{4 \pi \mu_{0}} \int_{s} \frac{B_{r}}{|\vec{r}|} d s \\
0
\end{array}\right.
$$

The permanent magnet is usually no free current. Permanent magnet field can be proved irrotational by electromagnetic theory and Maxwell equations. And magnetic field strength's seeking points can be expressed as the negative gradient of the magnetic bits as follows:

$$
\vec{H}=-\nabla U
$$

The derivative of the Gap in the space of a point:

$$
\nabla\left(\frac{1}{|\vec{r}|}\right)=\frac{\vec{r}}{|\vec{r}|^{3}}
$$

Substituting (8), (9) into (7) a permanent magnet eddy current coupling can be obtained in the air gap magnetic field intensity of a point as follows

$$
\vec{H}=\left\{\begin{array}{c}
-\frac{B_{r}}{4 \pi \mu_{0}} \int_{s} \frac{\vec{r}}{|\vec{r}|^{3}} d s \\
\frac{B_{r}}{4 \pi \mu_{0}} \int_{s} \frac{\vec{r}}{|\vec{r}|^{3}} d s \\
0
\end{array}\right.
$$

Further we can get permanent magnetic vortex coupling magnetic induction in the air gap of a point $\vec{B}$ as follows:

$$
\vec{B}=\mu_{2} \vec{H}
$$

Above among: $\mu_{2}$ is the permeability point in the air gap to be seeking.

\section{Permanent Magnetic Air Gap Magnetic Induction Eddy Current Coupling Strength Parameters Impact Analysis}

In order to enhance the air-gap magnetic flux density, we analyze the gap size, the thickness of the magnet, the magnetic radius and pole of the structural parameters. Select the copper coil average radius and circumferential position $30^{\circ}$, considering the impact of changes in the gap size, the thickness of the magnetic blocks, magnetic blocks radius and the number of poles of the air gap magnetic flux density in a single cycle. When using MATLAB portrayed as the size changes, the air gap magnetic flux density contrast curve, from the structure, the permanent magnet material cost we take increasing air-gap magnetic induction into account of the design program. The NdFeB is selected in twelve eddy current coupling pole permanent magnet as permanent magnet material. Its magnetic parameters, structural parameters are shown in Tables 1 and 2: 
Table 1. Neodymium Iron Boron (N35SH) Performance Parameters of Materials

\begin{tabular}{|c|c|c|c|c|}
\hline \multicolumn{2}{|c|}{ Residual magnetic induction intensity $\mathrm{Br}$} & \multicolumn{2}{|c|}{ Coercive force $H_{C B}$} & Working temperature $T_{W}$ \\
\hline 1. $294 T$ & & & $1592 K A / m$ & $80^{\circ} \mathrm{C}$ \\
\hline Relative permeability $u_{r}$ & \multicolumn{2}{|c|}{ Curie temperature $T_{C}$} & \multicolumn{2}{|c|}{ Maximum magnetic energy product $(\mathrm{BH})$ max } \\
\hline 1.05 & $300^{\circ} \mathrm{C}$ & & \multicolumn{2}{|r|}{$318 \mathrm{KJ} / \mathrm{m}^{3}$} \\
\hline
\end{tabular}

Table 2. Neodymium Iron Boron (N 35SH) Disk Structure Parameters

\begin{tabular}{|c|c|c|}
\hline Inside diameter $R 1$ & Outer diameter $R_{2}$ & Air gap $Z_{1}$ \\
\hline $90 \mathrm{~mm}$ & $140 \mathrm{~mm}$ & $5 \mathrm{~mm}$ \\
\hline Thickness $H$ & Pole center angle $a$ & The number of poles $N$ \\
\hline $20 \mathrm{~mm}$ & $30^{\circ}$ & 12 \\
\hline
\end{tabular}

\subsection{Effect of the Gap Size of the Air Gap Magnetic Induction}

When the thickness of the permanent magnet magnetic eddy current coupling block changes, the total strength of the radial component of the magnetic induction, the circumferential component and axial component also change. And the laws are consistent. We give an example of axial. The variation relation is shown in Figure 5-7. In Figure when the thickness of magnet size is changed from $10 \mathrm{~mm}$ to $30 \mathrm{~mm}$, with the increase of magnetic blocks thickness the axial component of the total magnetic flux density amplitude is gradually increasing. Increasing the thickness of magnet can be used within the appropriate range to increase the magnitude of the air gap magnetic flux density, thus to improve the performance of the permanent magnet eddy magnetic drive coupling.

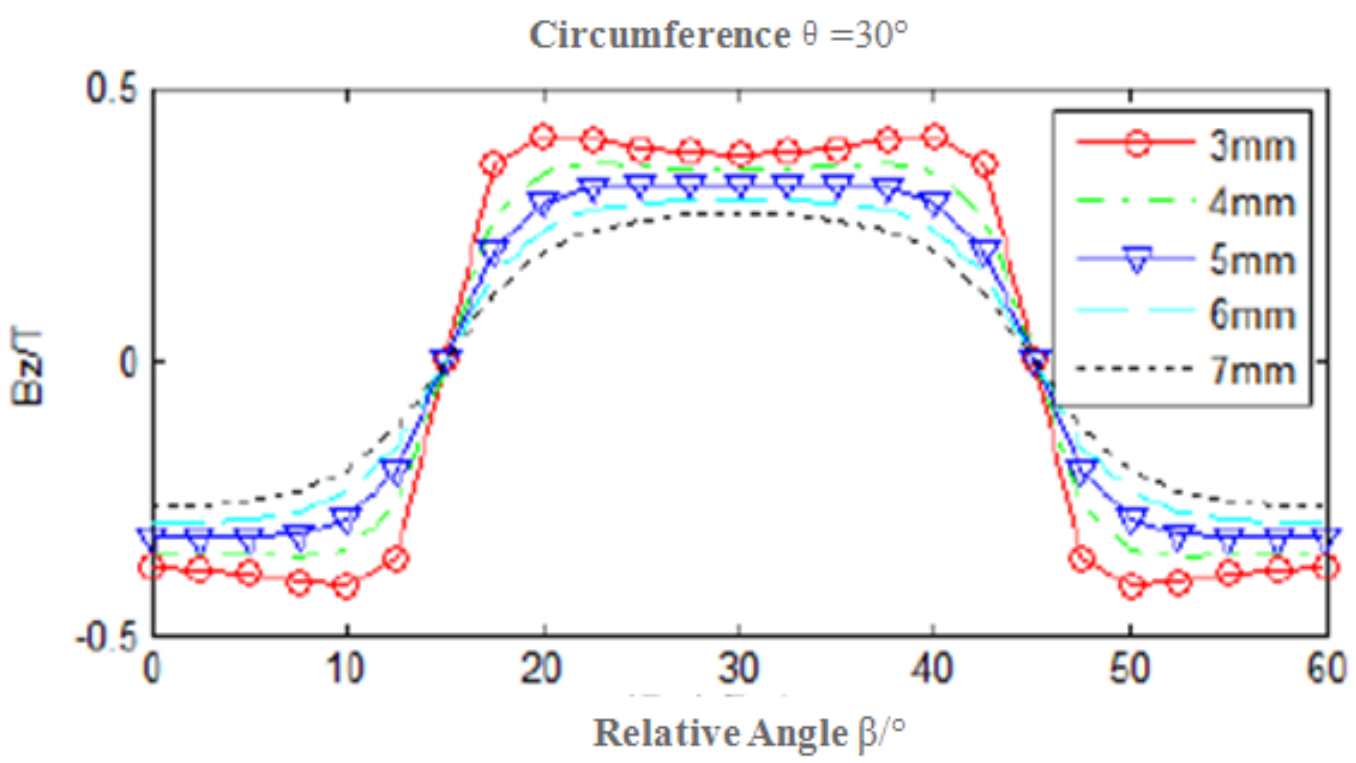

Figure 2. Gap Size Effect on the Axial Component of the Air-Gap Magnetic Flux Density Curve 


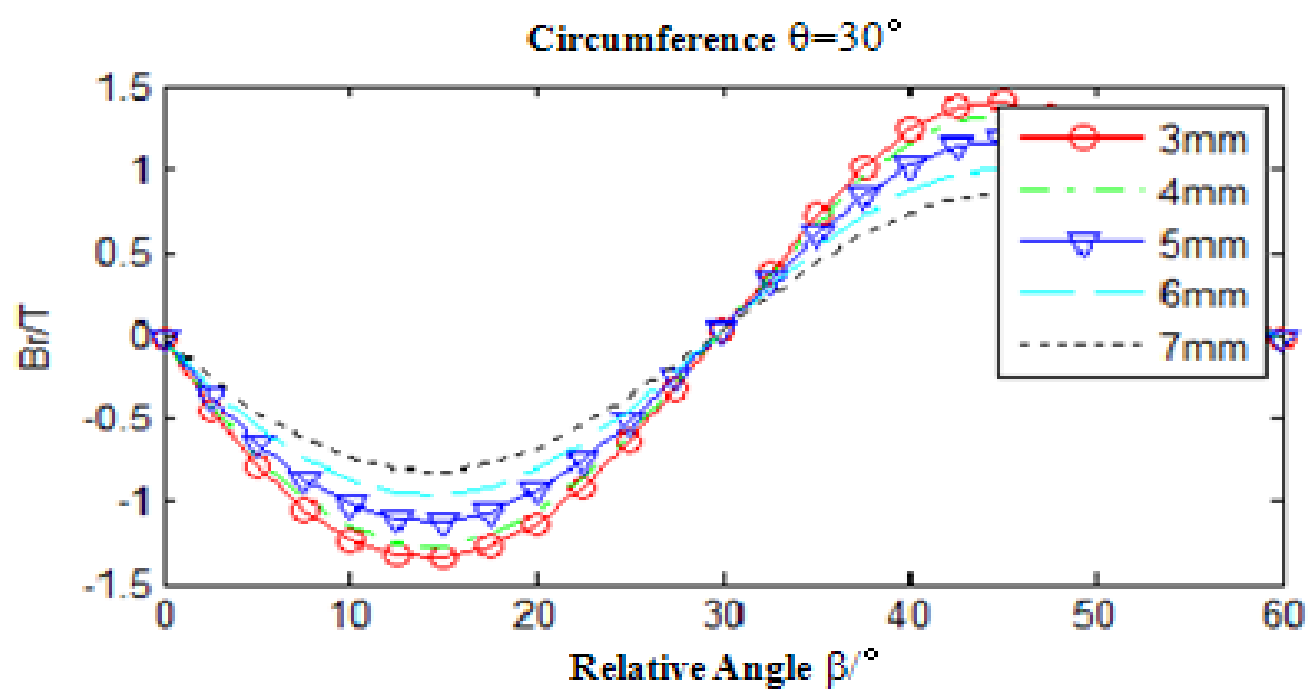

Figure 3. Gap Size Effect on the Radial Component of the Air-Gap Magnetic Flux Density Curve

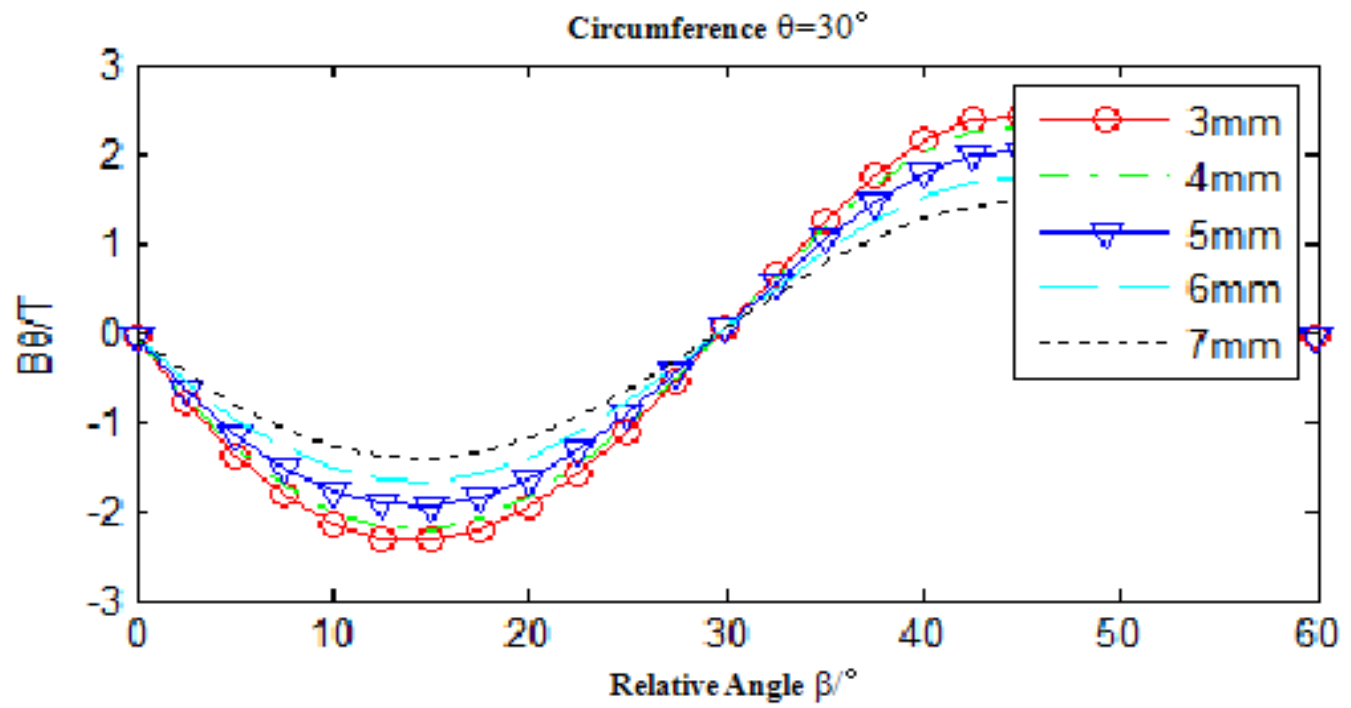

Figure 4. Gap Size Effect on the Circumferential Component of the Air-Gap Magnetic Flux Density Curve

\subsection{Affect the Thickness of the Air Gap Magnetic Block Magnetic Induction}

When the thickness of the permanent magnet magnetic eddy current coupling block changes, the total strength of the radial component of the magnetic induction, the circumferential component and axial component also change. And the laws are consistent. We give an example of axial. The variation relation is shown in Figure 5-7. In Figure when the thickness of magnet size is changed from from $10 \mathrm{~mm}$ to $30 \mathrm{~mm}$, with the increase of magnetic blocks thickness the axial component of the total magnetic flux density amplitude is gradually increasing. Increasing the thickness of magnet can be used within the appropriate range to increase the magnitude of the air gap magnetic flux density, thus to improve the performance of the permanent magnet eddy magnetic drive coupling. 


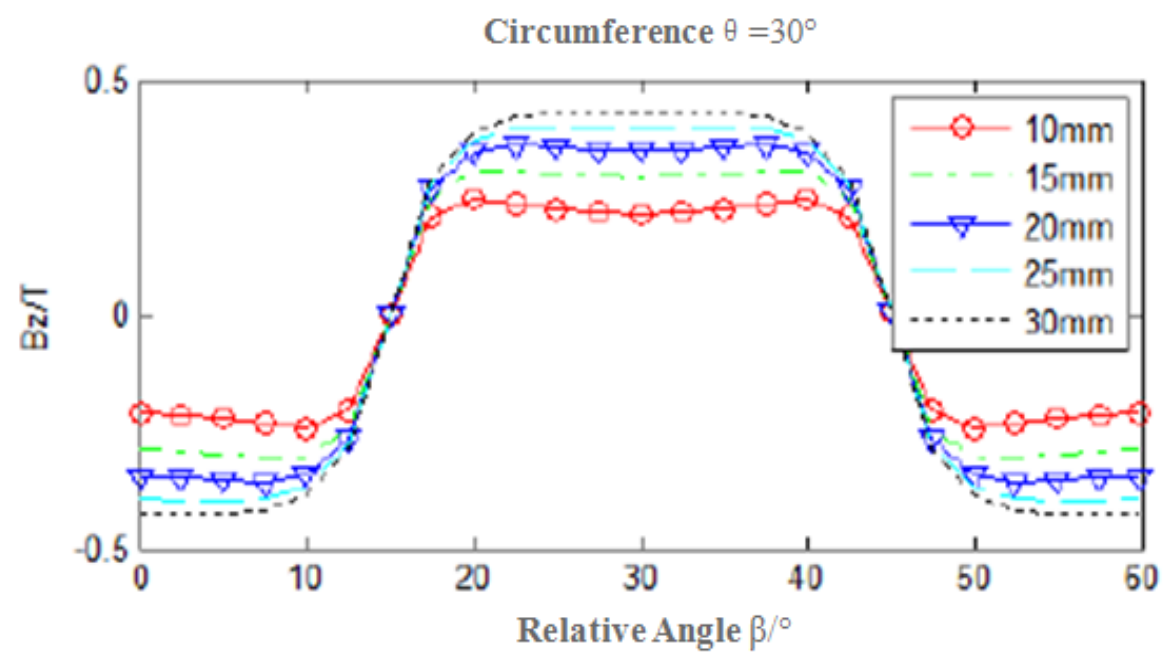

Figure 5. Magnet Thickness Effect on the Axial Component of the Air-Gap Magnetic Flux Density Curve

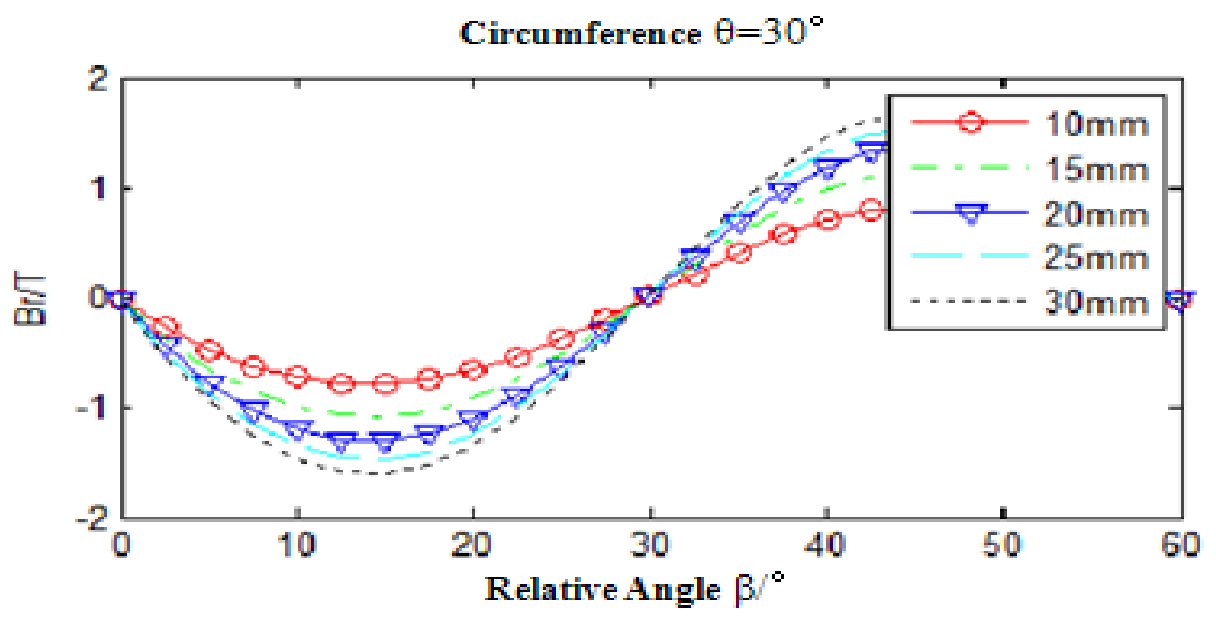

Figure 6. Magnet Thickness Effect on the Radial Component of the Air-Gap Magnetic Flux Density Curve

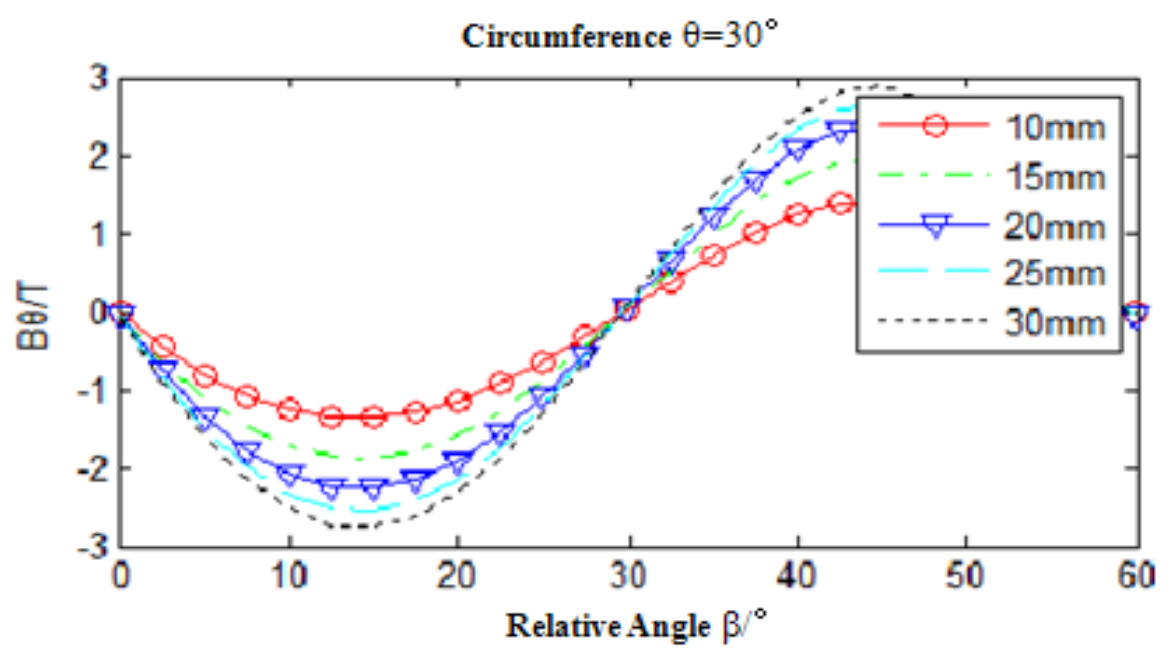

Figure 7. Magnet Thickness Effect on the Circumferential Component 


\subsection{Effect of Air-Gap Magnetic Block Radius of the Magnetic Induction}

When the magnetic block radius of permanent magnet eddy current couplings changes, the radial component, circumferential and axial components of the component of total magnetic induction all change and the laws are consistent. We give an example of axial. The variation relation is shown in Figure 8-10. In Figure when the thickness of magnet size is changed from $10 \mathrm{~mm}$ to $30 \mathrm{~mm}$, with the increase of magnetic radius, the magnitude of the total magnetic induction intensity is also increased. Although large changes happen in magnetic block radius, however, the magnitude of the magnetic flux density change is not obvious, so increasing the magnetic block radius on the magnitude of the magnetic flux density is not meaningful.

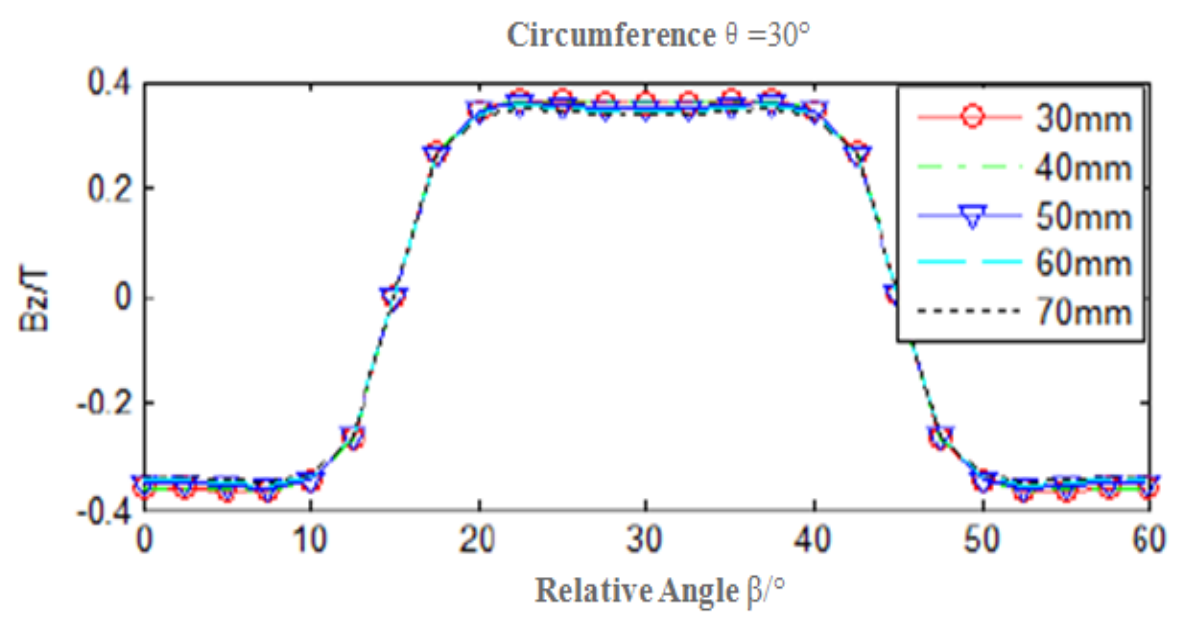

Figure 8. Block Radius of the Impact on the Air-Gap Magnetic Flux Density Axial Component of the Curve

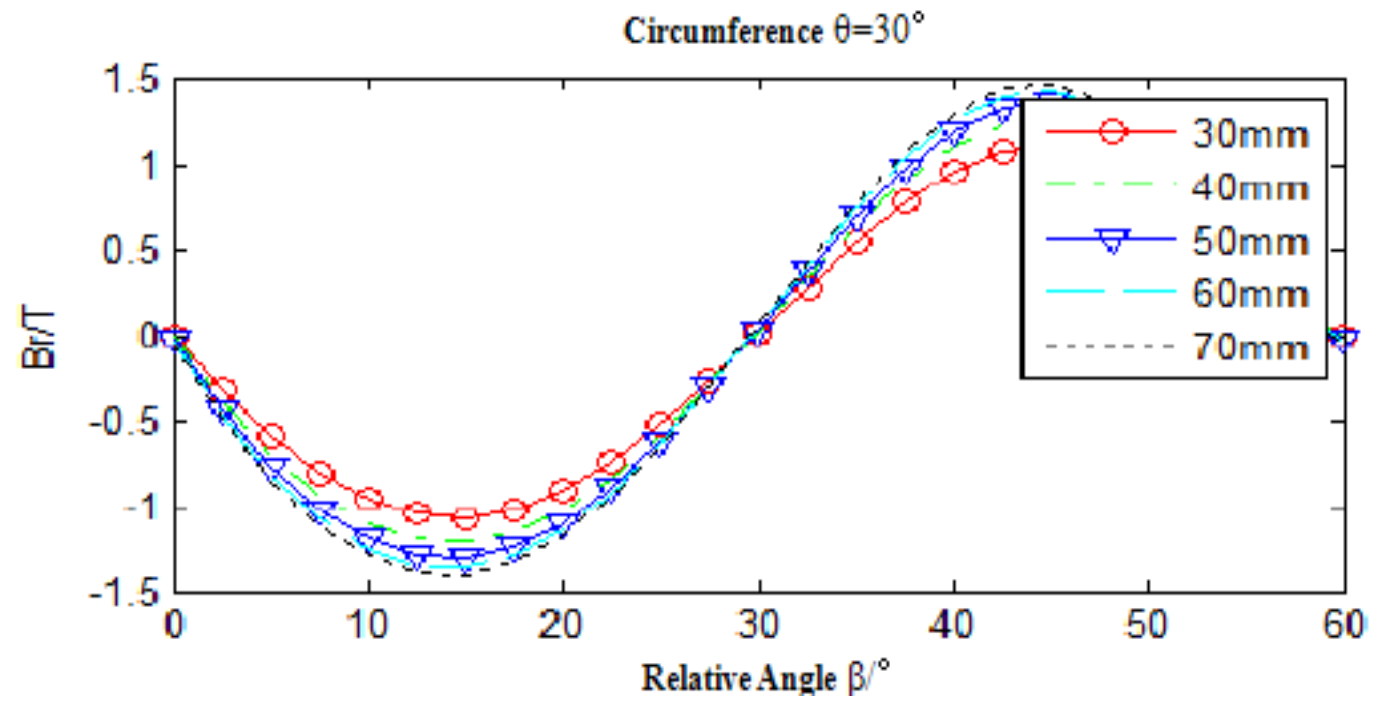

Figure 9. Block Radius of the Impact on the Air-Gap Magnetic Flux Density Radius Component of the Curve 


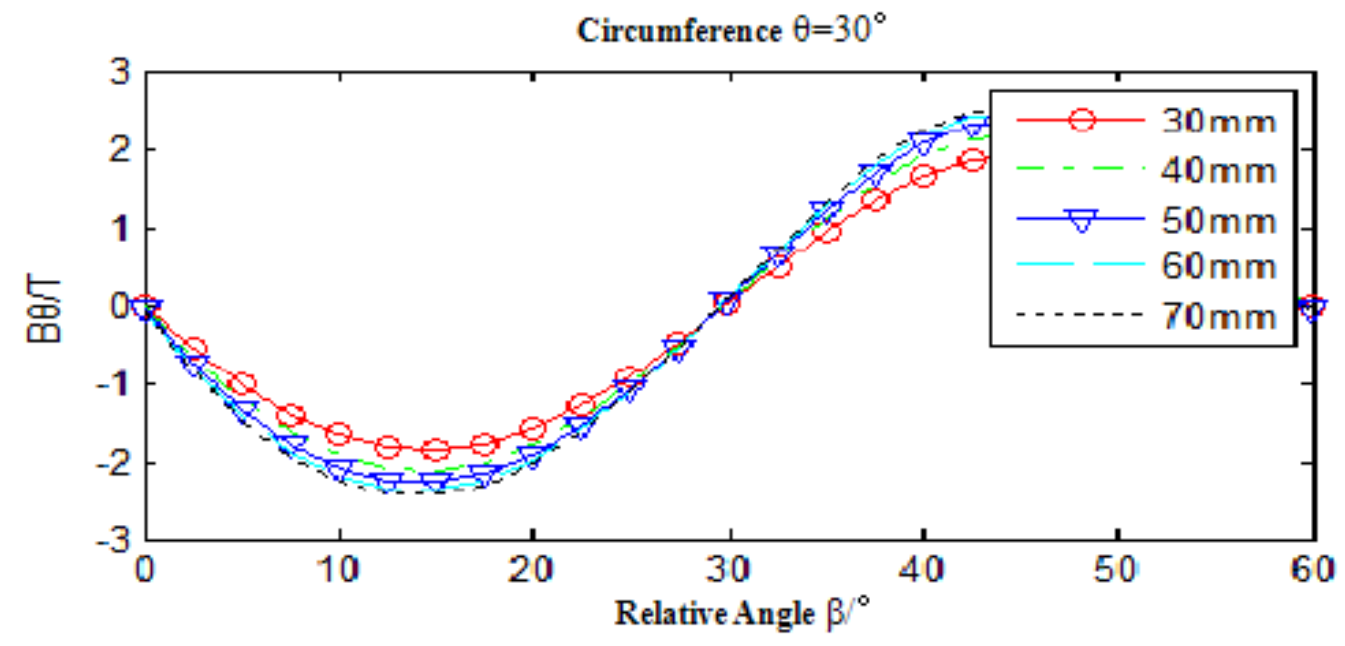

Figure 10. Block Radius of the Impact on the Air-Gap Magnetic Flux Density Circumferential Component of the Curve

\subsection{Effect of the Number of Poles of the Air-Gap Magnetic Induction}

When the permanent magnet poles Wing disk vortex coupling change, the Radial component, circumferential and axial components of the component of total magnetic induction all change and the laws are consistent. We give an example of axial. The variation relation is shown in Figure 11-13 in figure poles are increased from 8 to 16 with the increase of the magnetic poles, the axial component of the total magnetic flux density is slowly increased while the number of magnetic poles changes a lot. However, the magnitude of the magnetic flux density's increasing trend is not very clear. So choose a reasonable number of poles. You can reduce equipment costs at the same time when the amplitude of the magnetic flux density changes a little.

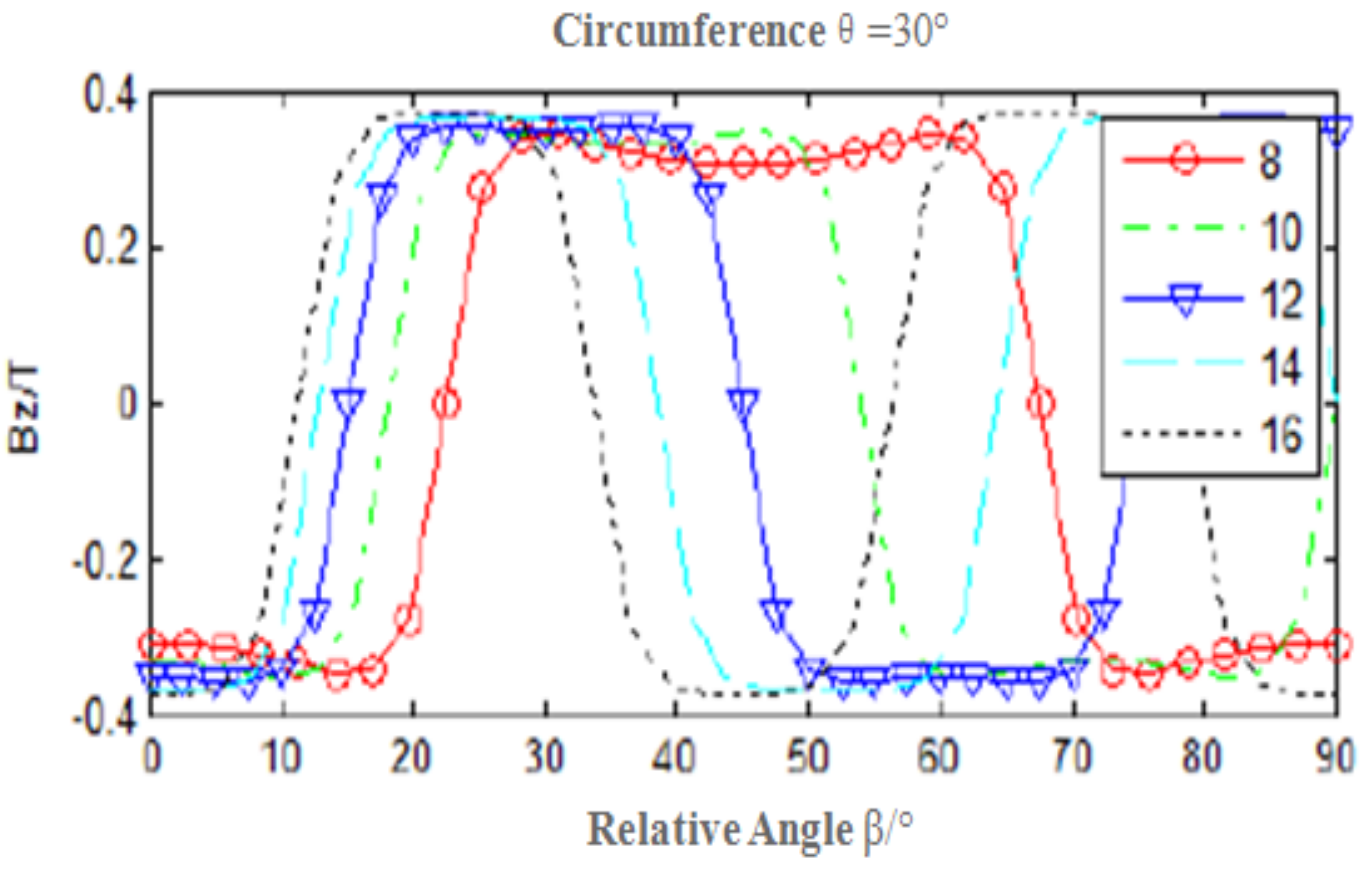

Figure 11. Poles on the Gap Axial Component of the Magnetic Induction Curves 


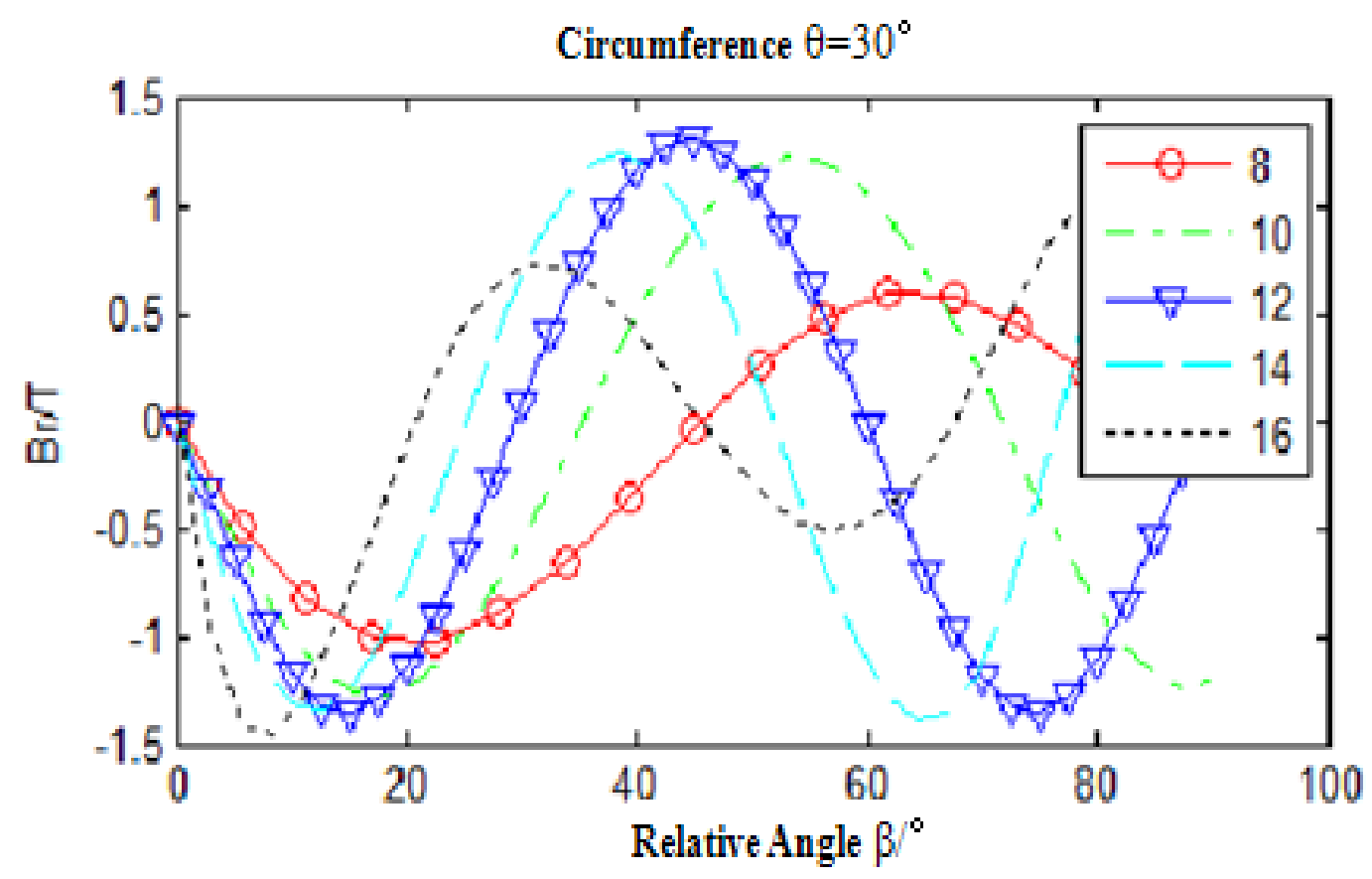

Figure 12. Poles on the Gap Radius Component of the Magnetic Induction Curves

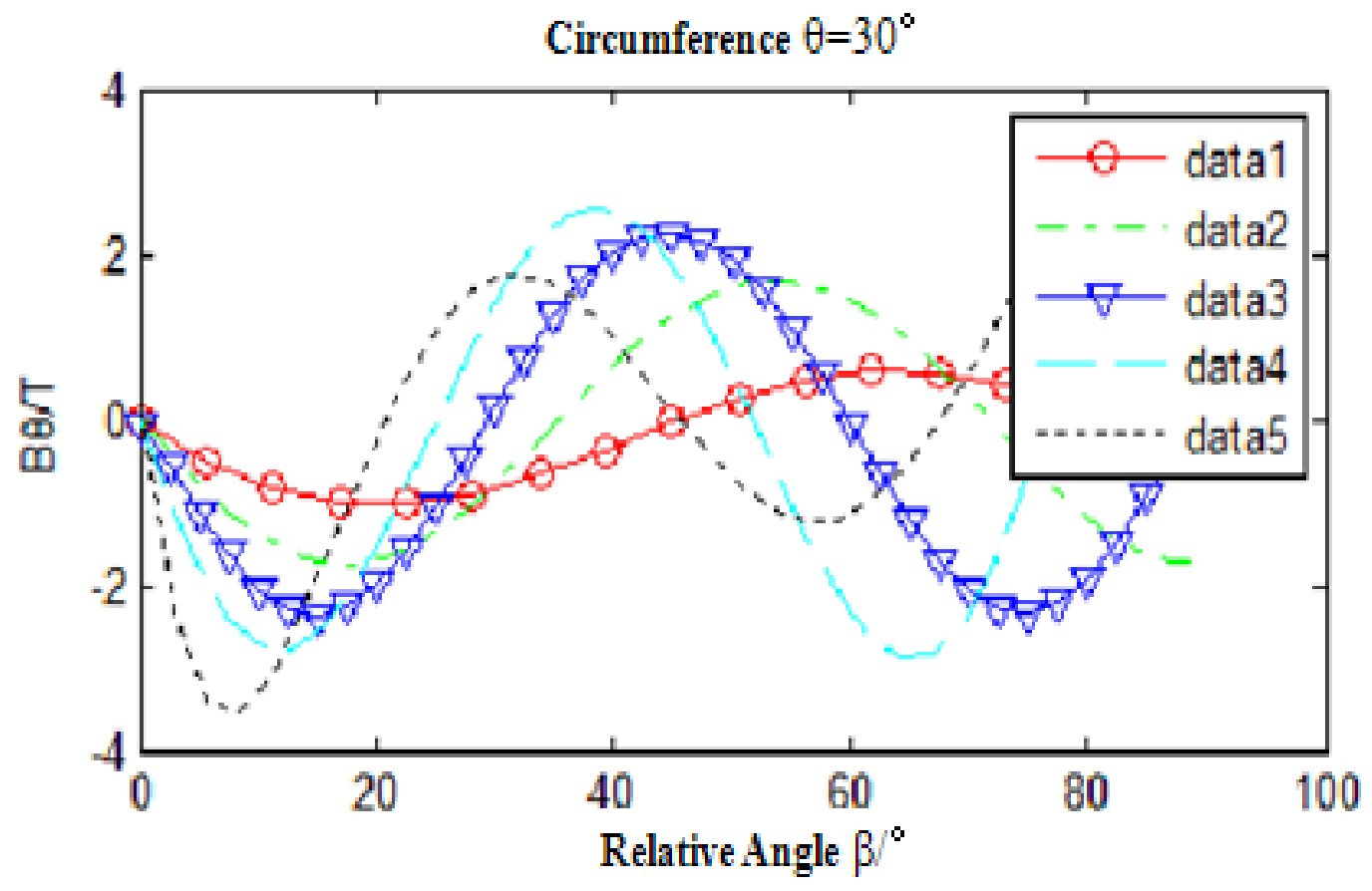

Figure 13. Poles on the Gap Circumferential Component of the Magnetic Induction Curves

\section{Conclusion}

It is mainly for the 12-pole permanent magnet eddy current couplings using the equivalent magnetic charge principle to establish an analytical mathematical model of the air-gap magnetic field, solving the air gap magnetic induction and using MATLAB to get the curve of parameters gap size, the thickness of the magnetic blocks and magnetic 
blocks radius's changeable effect on Air gap magnetic induction. Deficiency present in the analysis is that it does not consider the effect of the eddy current demagnetization and it only analyzes the distribution of the intensity of magnetic induction in the air gap of the permanent magnet only. The results show that: choose a smaller air gap distance within the allowable range, within a certain range by the way of increasing the thickness of the magnet block, selecting the reduced magnetic block radius within the appropriate range choosing a reasonable number of poles, can effectively increase the air-gap magnetic flux density amplitude aiming to reduce equipment costs. The air-gap magnetic flux density is analyzed in this chapter; establish the foundation for solving the distribution of eddy current density and magnetic torque transmission.

\section{Acknowledgments}

This work is supported by National Nature Science Foundation of China under Grant 61304069, 61372195, 61371200 and the Scientific Research Fund of Liaoning Provincial Education Department under Grant L2013496. Special thanks for above supports very much.

\section{References}

[1] A. Wallace, A. Jouanne and S. Williamson, "Performance prediction and test of adjustable, permanent-magnet, load transmission systems", IEEE Industry Applications Conference 36th IAS Annual Meeting, Chicago, (2001), pp. 1648-1655.

[2] A. Wallac, A. Jouanne and A. Ramme, "A permanent-magnet coupling with rapid disconnect capability", Proceedings of International Conference on Power Electronics Machines and Drives, Bath, (2002), pp. 286-291.

[3] C. A. Smith, A. Wakeel and A. Wallace, "Formal design optimization of PM drive couplings", IEEE Industry Applications Society Annual Meeting, Pittsburgh, (2002), pp. 205-211.

[4] H. Gholizad, M. Mirsalim and M. Mirzayee, "Motional eddy currents analysis in moving solid iron using magnetic equivalent circuits method", IEEE/ACES International Conference on Wireless Communications and Applied Computational Electromagnetics, Honolulu, (2005), pp. 535-538.

[5] Y. Peng, Q. J. Ruan and Y. Zhang, "A composite grid method for moving conductor eddy current problem", IEEE Transactions on Magnetics, vol. 43, no. 7, pp. 3259-3265.

[6] A. Wallace, C. Wohlgemuth and K. Lamb, "A high efficiency, alignment and vibration tolerant, copler using high energy-product permanent magnets", Seventh International Conference on Electrical Machines and Drives, Durham, (1995), pp. 232-236.

[7] A. Wallace and A. Jouanne, "Industrial speed control: are PM couplings an alternative to VFDs", IEEE Industry Applications Magazine, vol. 7, no. 5, (2001), pp. 57-63.

[8] A. Wallace, A. Jouanne and R. Jeffreys, "Comparison testing of an adjustable-speed permanent-magnet eddy current coupling", Proceedings of IEEE Pulp and Paper Conference, Atlanta, (2000), pp. 73-78.

[9] X. Wang and D. Z. Wang, "Magnetic circuit structural design of Permanent Magnetic Drive", Electric Drive, vol. 41, no. 10, (2011), pp. 55-58.

[10] X. Wang, D. Z. Wang and Z. Liu, "Eddy current field analysis and performance calculations for adjustable permanent magnetic coupler", Chinese Journal of Scientific Instrument, vol. 33, no. 01, (2012), pp. 155-160.

[11] X. Wang and D. Z. Wang, "Calculation of eddy current loss and thermal analysis for adjustable permanent magnetic coupler", International Conference on Electronic and Mechanical Engineer and Information Technology, (2011).

[12] Y. Zhang, H. Zhou and Z. H. Sun, "Research of the orthoscopic permanent magnet eddy current damper in magnetic stage", International Conference on Computer Application and System Modeling, (2010).

[13] J. Alexandrova, H. Jussila, J. Nerg and J. Pyhonen, "Comparison between models for eddy current loss calculations in rotor surface-mounted permanent magnets", ICEM International Conference on Electrical Machines, (2010).

[14] B. X. Zhao and Y. C. Ji, "Analysis of flux leakage in novel permanent magnet type eddy current retarder for vehicle applications", IEEE Vehicle Power and Propulsion Conference, (2008) September 3-5.

[15] G. Cabiva, "Optimization of radial eddy current couplings", The International Journal for Computation and Mathematics in Electrical and Electronic Engineer-ing, vol. 24, no. 3, (2005), pp. 767-783.

[16] K. Hamideh, M. Razavi and U. Lamperht, "Eddy-coupling with slotted conductor disk", IEEE Transactions on magnetic, vol. 42, no. 3, (2005) March, pp. 405-410.

[17] Y. Katsumi, "Loss analysis of interior permanent magnet motors considering carrier harmonics and magnet eddy currents using 3-D FEM", IEEE Transactions on Magnetic, vol. 40, no. 2, (2007) May. 
[18] S. Ekram, D. Mahajan, M. Fazil, V. Patwardhan and N. Ravi, "Design optimization of brushless permanent magnet hub motor drive using FEA", Proceeding of International Conference on Electrical Machines and Systems, (2007), October 8-11.

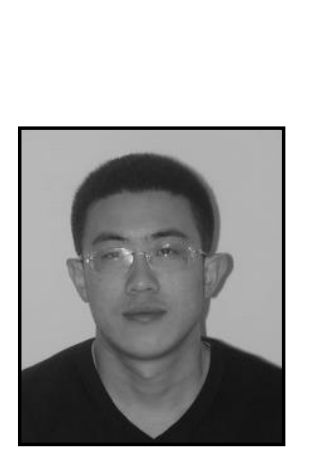

\begin{abstract}
Authors
Qingzhong Gao, received his B.Sc. from Northeastern University in 2005, and received his M. Sc. from Northeastern University in 2009. Now he is a doctoral candidate in Northeastern University. His main research interest is key technologies of adjustable permanent magnetic coupler. His research interests include Speed Permanent Magnet key technologies.
\end{abstract}

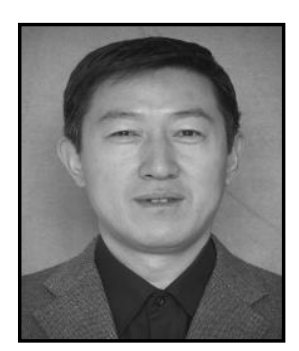

Dazhi Wang, is a professor and doctoral supervisor in Northeastern University, mainly engaged in the research of electric power system and electric power transmission.

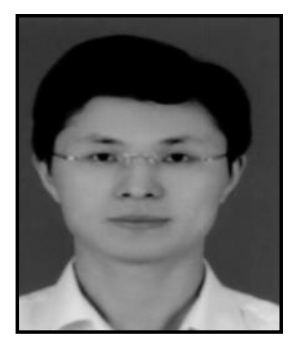

Sheng Lin, has received his B.Sc. from Northeastern University in 2005, and received his M. Sc. from Northeastern University in 2009. Now he is a doctoral candidate in Northeastern University. His main research interest is key technologies of adjustable permanent magnetic coupler. His research interests include Speed Permanent Magnet key technologies. 